\title{
Left ventricular myocardial dysfunction secondary to adverse ventricular-ventricular interactions in previously healthy infants with Respiratory Syncytial Virus Bronchiolitis
}

\author{
Moises Rodriguez-Gonzalez ${ }^{1}$ \\ ${ }^{1}$ Affiliation not available
}

October 26, 2020

Article

Title: Left ventricular myocardial dysfunction secondary to adverse ventricular-ventricular interactions in previously healthy infants with Respiratory Syncytial Virus Bronchiolitis...

Moises Rodriguez-Gonzalez 1,4*, Alvaro Antonio Perez-Reviriego1,4, Ana Castellano-Martinez2,4, Simon Lubian-Lopez3,4 and Isabel Benavente-Fernandez3,4

1 Pediatric Cardiology Division, Puerta del Mar University Hospital, Cadiz, Spain;doctormoisesrodriguez@gmail.com;alvaro.apr@hotmail.com

2 Pediatric Nephrology Division, Puerta del Mar University Hospital, Cadiz, Spain;anacastellanomart@gmail.com

3 Neonatology Division, Puerta del Mar University Hospital, Cadiz, Spain;isabenavente@gmail.com;slubian@yahoo.es

4 Biomedical Research and Innovation Institute of Cadiz (INiBICA), Research Unit, Puerta del Mar University Hospital

* Correspondence: doctromoisesrodriguez@gmail.com.

Received: date; Accepted: date; Published: date

Abstract: Aim: To investigate if the presence of left ventricular myocardial dysfunction (LVMD) assessed by Tei index (LVTX) may have a direct impact on the outcomes in Respiratory Syncytial Virus bronchiolitis (RSVB), and if NT-proBNP will increase the accuracy of traditional clinical and laboratory markers in predicting the severity of the disease. Methods: A single-centre, prospective, cohort study including healthy infants aged 1-12 month-old admitted due to RSVB between October 1, 2016 and April 1, 2017. All patients underwent clinical, laboratory and echocardiographic evaluation within 24 hours of admission. PICU admission was defined as severe disease. Results: We enrolled 50 cases of RSVB (median age of 2 (1-6.5) months; $40 \%$ female) and 50 age-matched controls. We observed higher values of LVTX in infants with RSVB than in controls (0.42 vs $0.36 ; \mathrm{p}=0.008)$. A total of $9(18 \%)$ cases presented LVMD (LVTX $>0.5)$, with higher incidence of PICU admission ( $89 \%$ vs $5 \%$; $<<0.001)$. The diagnostic performance of NTproBNP to predict LVMD in infants with RSVB resulted high (AUC 0.91 (CI95\% 0.79-0.98). The diagnostic yield of the predictive model for PICU admission that included NTproBNP was excellent (AUC 0.945, CI95\% 0.880-1), and significantly higher than the yields for models without NTproBNP. Conclusions: LVMD could be present in healthy infants with RSVB, negatively impacting the outcome. NTproBNP seems to be an adequate biomarker for LVMD and subsequently outcome. 
Keywords: Respiratory Syncytial Virus; NT-proBNP; Echocardiography; Pulmonary hypertension; Myocardial dysfunction; Tissue Doppler Imaging; Tei Index; Biomarkers; Infants.

\section{Introduction}

Respiratory Syncytial Virus bronchiolitis (RSVB) is the leading cause of lower respiratory infection and hospital admission among children up to 2 years of age worldwide [1]. Approximately 2-6\% cases of RSVB will develop a severe form of disease, requiring ad mission at the pediatric intensive care unit (PICU) and mechanical ventilation (MV) [1,2]. RSVB constitutes approximately $13 \%$ of all PICU admissions [2]. Current guidelines recognise identification of specific risk factors (congenital heart disease (CHD), chronic lung disease (CLD), prematurity, etc.) and clinical evaluation as the best tools to asses severity, predict evolution and tailor management [3].

Cardiovascular involvement seems to be a relevant prognostic factor in RSVB. Cardiovascular complications, are present in up to $9 \%$ of cases of RSVB, and constitute the second most common extra pulmonary manifestations after infectious complications [4]. These events present usually in an abrupt and unexpected manner in those children with severe RSVB, and infants with CHD are particularly susceptible to have these complications and adverse outcomes [5]. Interestingly, nearly half of children admitted to PICU with RSVB are healthy prior to the clinical event [2]. In these patients the presence of acute lung injury secondary to RSVB can also lead to important cardiovascular effects, especially raising pulmonary vascular resistance and overloading the right ventricle (RV) [6-8]. Moreover, previous studies assessing plasmatic levels of cardiac troponin in RSVB suggest an underrecognized but yet clinically significant incidence of myocardial damage in this population [9-11]. Furthermore, RV global dysfunction in ventilated healthy infants has been reported [12]. Recently, we found that mild to moderate forms of PH could impact the outcome of healthy infants with RSVB [13].

Adverse RV-LV interactions and left ventricle (LV) myocardial dysfunction (LVMD) are emerging as important determinants of PH outcomes. PH can induce complex changes in LV geometry and causes an abnormal relaxation and a non-uniform contraction pattern in the LV wall, leading to LVMD [14-16]. However, most studies in healthy infants with RSVB found no abnormalities when assessing LVMD through conventional echocardiographic parameters [12,13,17-19]. Remarkably, there are no studies to date assessing LVMD in RSVB by more sensitive methods such as tissue Doppler imaging (TDI) echocardiography.

$\mathrm{N}$-terminal pro-B-type natriuretic peptide (NT-proBNP) is a hormone synthesized and released into the circulation by ventricular myocytes in response to pressure/volume overload and an increase in myocardial wall stress [20]. Elevated serum NT-proBNP levels have been defined as a powerful biomarker in the diagnosis of PH, and both LVMD and RV myocardial dysfunction (RVMD) secondary to pulmonary diseases [21-25]. Of note, we recently showed how NTproBNP could be considered an adequate biomarker for PH in previously healthy infants with RSVB [13].

In this study we aimed to investigate the adverse RV-LV interactions and LVMD through TDIechocardiography in previously healthy infants with RSVB. We hypothesized that acute PH with RV pressure overload may indeed have a direct impact on LV performance. We also hypothesized that those infants with LVMD are prone to develop a more severe form of disease. Finally, we sought to test NT-proBNP as a biomarker for LVMD, and to explore if NT-proBNP will increase the accuracy of traditional clinical and laboratory markers in predicting the severity of the disease.

2. Materials and Methods

\subsection{Design, settings and study population:}

This was a single-centre, prospective, cohort study including infants aged 1-12 month-old admitted to the Pediatric Department of our institution (a tertiary university-affiliated hospital in Spain) due to RSVB (determined by a confirmed RSV antigen testing) between October 1, 2016 and April 1, 2017. All patients underwent clinical, laboratory and echocardiographic evaluation within 24 hours of admission. We excluded patients with co-existing CHD or CLD, prematurity, those that received MV or intravenous fluid before 
assessment, and those with poor quality echocardiographic images or incomplete medical records. Severe cases were screened for coinfection and if existed they were also excluded. The control group consisted of age-matched healthy infants who underwent evaluation for heart murmur at our Pediatric cardiology outpatient clinic during the study period. These controls followed the same echocardiographic protocol as study patients. Our Institutional Review Board approved the study. Informed consent was obtained for all patients.

\subsection{Clinical and laboratory assessment and outcomes:}

The bronchiolitis score of Sant Joan de Déu (BROSJOD) [26]was used to assess severity at admission clinically. A BROSJOD score greater than 10 points is indicative of severe clinical state. Venous $\mathrm{pH}$ and $\mathrm{pCO} 2$ were determined, and respiratory acidosis (RA) was considered when $\mathrm{pH}<7.35$ and $\mathrm{pCO} 2>45 \mathrm{mmHg}$ coexisted in the same patient. Plasma NT-proBNP levels at admission were determined using a commercially available electrochemiluminescent immunoassay kit (ElecSys 2010, Roche Diagnostics). The primary outcome was PICU admission during the hospitalization. PICU admission criteria for RSVB at our institution rely on the presence of: apnea, extreme bradycardia, need of respiratory support greater than high-flow nasal cannula oxygen therapy, or inotropic support.

\subsection{Echocardiographic assessment:}

Standard techniques to obtain M-mode, two-dimensional and Doppler (color, pulsed, continuous and TDI) echocardiograms were performed by the same experienced pediatric cardiologist (RG.M) as recommended in the guidelines for the pediatric echocardiogram[27]. Images were obtained using Phillips IE33 ultrasound scanner with an 8 or $12 \mathrm{MHz}$ sectorial transducer. Each examination was recorded, and all of the studies were reviewed off-line by 2 observers (RG:M \& PR.A), who were blinded to the patient's clinical profile. All echocardiographic measurements represent the average of 3 beats. Control and case echocardiographic data were deidentified before data analysis.

\subsubsection{Right-sided echocardiographic assessment:}

The RV end-diastolic diameter (RVEDD) and the RV/LV ratio (RVLVr) were used as indicators of RV dilation. RV systolic function was assessed by the tricuspid annular plane systolic excursion (TAPSE) [28], and by the tissue Doppler imaging (TDI) derived peak systolic annular velocity at the tricuspid level of the RV free wall (St). RV Tei index (RVTX) was used as a measurement of global (systolic and diastolic) RV function. It was calculated using the TDI-derived isovolumic contraction (IVC), isovolumic relaxation (IVR) and ejection time (ET) intervals (measured at the lateral part of the tricuspid annulus) as previously described[29]. RV systolic pressure was calculated by the systolic gradient of the tricuspid regurgitation jet (TRJG) and using the simplified Bernoulli equation [30]. Due to the absence of an adequate tricuspid regurgitation jet in most patients, the RV outflow tract acceleration time/ejection time ratio (ATET) [31], the LV systolic eccentricity index (LVEI) [32], and the presence of septal flattening at the end of systole on the qualitative assessment were also used as indicators of RV systolic pressures [30]. The LVEI was also used as a measurement of leftward displacement of the interventricular septum (IVS).

\subsubsection{Left-sided echocardiographic assessment:}

The LV end-diastolic diameter (LVEDD) was used as indicator of LV dilation. The LV shortening fraction (LVSF) and the tissue Doppler imaging (TDI)-derived peak systolic annular velocity at the mitral level of the LV free wall $(\mathrm{Sm})$ were used to assess LV systolic function. To assess the diastolic LV function we obtained the mitral peak early (E) and late (A) diastolic velocity using pulsed wave Doppler of the mitral valve inflow. Also, TDI-derived early diastolic mitral annulus velocity (Em) was measured at the lateral part of the mitral annulus. The E/Em ratio and E/A ratio were calculated as indicators of LV filling pressures. LV Tei index (LVTX) was used as a measurement of global (systolic and diastolic) LV function. It was calculated using the TDI-derived isovolumic contraction (IVC), isovolumic relaxation (IVR) and ejection time (ET) intervals (measured at the lateral part of the mitral annulus) as previously described[29]. Cui \& Robertson reported in 2006 that the mean normal value of the LVTX for infants aged 1-12 months is 0.35 (0.09), and that a 
LVTX less than 0.5 is the upper limit of normal (2Zscore) [33]. Therefore, a LVTX $>0.5$ was defined as LVMD in this study. Figure $\mathbf{1}$ and Figure $\mathbf{2}$ show the main echocardiographic measures used in this study.

\subsection{Reproductibility:}

To explore intra-observer and inter-observer agreement, 30 echocardiographic studies were randomly selected and analysed offline. To estimate the intra-observer agreement, the first observer (RG.M) remeasured the LVTX, RVTX and LVEI with a 30-days interval blinded to previous measurements and patient information. To assess the inter-observer agreement, the means of both observers for each measurement were compared.

\subsection{Statistical analysis:}

Continuous data are presented as median (range) or mean (standard deviation) after testing for normality with the Shapiro-Wilk test; categorical data are presented as frequencies and percentage. Mean comparison was performed using Student's t test or Wilcoxon Mann-Whitney test as appropriate. Proportions were compared using Chi-square test or exact methods as necessary. Pearson and Spearman coefficients were used to assess correlations between continuous data. A receiver operator curve (ROC) analysis was used to determine the diagnostic accuracy of NTproBNP for LVMD. The best cut-off value of NT-proBNP to detect LVMD was empirically estimated based on the Liu method, and values of sensitivity (Se), specificity (Sp), negative predictive value (NPV) and positive predictive value (PPV) were calculated for the obtained cut point. Prediction models were developed using multivariate logistic regression analysis to identify potential predictors of PICU admission. The selection of the included variables was based on the theoretical background and the exploratory analysis. The discriminating ability of each model was assessed by the area under the receiver operator characteristic (AUC) curve. The AUCs from the obtained models were then compared by using the DeLong method[34] to determine whether any model resulted in increased predictive accuracy. The reliability of echocardiographic measurements was evaluated with the intra-class correlation (ICC) coefficients and Bland-Altman (BA) analysis[35]. Based on the 95\% confident interval of the ICC coefficients, values less than 0.5 , between 0.5 and 0.75 , between 0.75 and 0.9 , and greater than 0.90 were considered indicative of poor, moderate, good, and excellent reliability, respectively. All the statistical analyses were performed using the Stata 13.0. (StataCorp. 2013. Stata Statistical Software: Release 13. College Station, TX: StataCorp LP.). A P value $<0.05$ was considered statistically significant.

3. Results

3.1. Baseline characteristics and outcomes of patients with RSVB: We enrolled a total of 50 cases of RSVB with a median age of 2 (1-6.5) months (40\% female). The control group consisted of 50 healthy infant with no differences regarding age, sex or BSA distribution (Table 1). RSVB patients were admitted 2.76 (1.23) days after the initial symptoms, with a median BROSJOD score of 6 (1-14), a median SpO2 of $93 \%(87-98 \%)$, and a median heart rate of $118 \mathrm{bpm}$ (89-179 bpm). Up to 9 (18\%) cases presented RA and a BROSJOD score $>10$. A total of $10(20 \%)$ cases needed PICU admission within 1.20 (0.38) days from hospitalization (length of PICU stay 5 (2-9) days), and were classified as having a severe RSVB. 3 of these10 cases required MV and 7 of them required CPAP/BiPAP. No cases of arrhythmia different from sinus tachycardia were observed. No patient required inotropic support and none of the included patients died.

3.2. Echocardiographic alterations in patients with RSVB: The RSVB group had a higher proportion of pericardial effusion ( $34 \%$ vs $6 \% ; \mathrm{p}<0.001)$. All cases of pericardial effusion were mild and did not require any treatment. We observed higher values of LVTX (0.42 vs $0.36 ; \mathrm{p}=0.008)$ in infants with RSVB than in controls(Figure 3). The RSVB group also presented more cases of septal flattening (28\% vs 6\%; $\mathrm{p}=0.003$ ), and higher TRJG (27 vs $22 \mathrm{mmHg} ; \mathrm{p}=0.013)$, RVTX (0.39 vs $0.36 ; \mathrm{p}=0.005)$ and LVEIs (1.08 vs $1 ; \mathrm{p}<0.001)$ than the control group. There were no differences between RSVB and control groups regarding echocardiographic parameters of ventricular dimensions, systolic or diastolic function (Table 1).

3.3. Adverse LV-RV interactions in patients with RSVB:In the RSVB group, increased LVTX was related to echocardiographic parameters indicating higher RV dimensions (Rho RVDD $=0.56$, Rho RVLVr 
$=0.60$ ), higher RV pressures $($ Rho $\mathrm{TRJG}=0.54$, Rho ATET $=-0.50$, Rho LVEI $=0.77$ ), and decreased RV global function (Rho RVTX $=0.74$ ). We did not find associations of echocardiographic measurements of RV systolic function (TAPSE and St) with LVTX (Table 2 \& Figure 4).

3.4. LVMD in patients with RSVB: We found LVMD, defined by a LVTX $>0.5$, in 9 (18\%) patients with RSVB (Table 3). LVMD was associated to PICU admission (89\% vs 5\%; $\mathrm{p}<0.001)$. These patients presented higher BROSJOD scores (11 vs 6; p<0.001), lower SpO2 (90\% vs 94\%; p <0.001), more cases of RA ( $55 \%$ vs $9 \% ; \mathrm{p}=0.001)$, and higher NT-proBNP levels $(2221 \mathrm{pg} / \mathrm{ml}$ vs $377 \mathrm{pg} \mathrm{ml} ; \mathrm{p}<0.001)$ than those with a normal LV myocardial function. The LVTX was also strongly correlated with NT-proBNP levels (Rho $=0.73$ ) (Figure 5).

3.5. NTproBNP as biomarker for LVMD: The diagnostic performance of NTproBNP to predict LVMD in infants with RSVB resulted high with an area under the ROC curve of 0.91 (CI95\% 0.79-0.98) (Figure 6). The best estimated cut-off value to predict LV myocardial dysfunction on echocardiography was 1500 $\mathrm{pg} / \mathrm{ml}$, that correctly classified $92 \%$ of cases, and presented a Se of 0.80 (CI95\% 0.49-0.94), Sp of 0.95 (CI95\% 0.83-0.98), a PPV of 0.80 (CI95\% 0.49-0.94), and a NPV of 0.95 (CI95\% 0.87-0.99) (Youden index $0.75)$.

3.6. Clinical and laboratory predictors of PICU admission in RSVB: We developed 3 different predefined prediction models for PICU admission in RSVB. The variables used were clinical and laboratory parameters that are usually used to assess severity in RSVB (age, clinical score and RA), and NT-proBNP as biomarker for LVMD. Model 1 included age $<3$ months, BROSJOD score $>10$, RA, and NTproBNP $>$ $1500 \mathrm{pg} / \mathrm{ml}$. Model 2 included age $<3$ months, BROSJOD score $>10$, and RA. The model 3 included age $<3$ months and BROSJOD score $>10$. The diagnostic yield of model 1 for PICU admission was excellent (AUC 0.945, CI95\% 0.880-1), and significantly higher than the yields for model $2(\mathrm{p}=0.033)$ and model 3 $(\mathrm{p}=0.026)$. There were no differences between ROC curves of models 2 and $3(\mathrm{p}=0.432)$. (Table 4 \& Figure 7 ). In model 1, the presence of NT-proBNP levels $>1500 \mathrm{pg} / \mathrm{ml}$ was the only independent predictive factor for PICU admission in RSVB, with an OR 27.03 (CI95\% (1.50-487), p=0.025).

\section{Discussion}

The main finding of our study is that LVMD was observed at early stages of the disease in up to $18 \%$ of previously healthy infants with RSVB when assessed by DTI-derived LVTX. The LVMD was associated with a worse respiratory state, PICU admission, and echocardiographic signs of RV pressure overload and RVMD, indicating the presence of adverse RV-LV interactions in cases of severe RSVB. Also, we observed that NTproBNP identified accurately LVMD. Moreover, we found an added benefit to the addition of NTproBNP to the clinical evaluation in predicting the development of a severe disease in this population.

CHD are important causes of morbidity and mortality in RSVB[5]. This may be related to multiple physiological factors including baseline compromised cardiorespiratory function and the potential development of PH. However, little is known about LVMD and its association with RV function, pulmonary hemodynamics, and outcomes in previously healthy infants with RSVB. In accordance with literature, the present study reveals that conventional parameters of myocardial function are not altered in RSVB [17-19]. Only one previous study has assessed myocardial performance using the TEI index in RSVB [12]. Our results are consistent with those reported by Thorburn et al. who found a RVMD in ventilated patients with severe RSVB. However, they did not demonstrate PH as the cause of RVMD. This could be due to the use of only TRJG as echocardiographic marker of RV pressures, and the presence of PH might had been underestimated. In a recent work from our group we used a combination of different echocardiographic parameters to assess RV pressures, $\mathrm{PH}$ was reported in up to $22 \%$ of RSVB cases at early stages of the disease, but accordingly with Thorburn et al, we did not found associations between PH and RV or LVMD [13]. Remarkably, we assessed the ventricular function only by conventional parameters (TAPSE and LVSF).

To the best of our knowledge, this is the first study to evaluate LVMD using TDI-derived LVTX, and to assess RV-LV interactions in infants with RSVB. LVTX, which includes both systolic and diastolic time intervals to assess the global cardiac dysfunction, is an easily performable, recordable and reproducible parameter 
with normal reference values that can be applied to the entire spectrum of the paediatric population, with no impact of age, heart rate, and BSA[36]. Using LVTX we found a LVMD in near $20 \%$ of cases. The RV shares muscle fibers, the inter-ventricular septum (IVS), and the pericardial sac with the LV. Consequently, changes in RV affect the LV, a concept termed ventricular interdependence $[16,37,38]$. In this study, we observed a moderate to strong correlation between LVTX and leftward displacement of the IVS (LVEI), raised RV pressures (TRGJ, ATET) and reduced RV global function (RVTX), supporting our hypothesis that through ventricular interdependence, RV pressure overload and RV dysfunction could induce LVMD in RSVB. These observations could add new insights into the pathophysiology of RSVB, highlighting a key role of the cardiovascular system, especially LV myocardial performance in this setting. Should our findings be validated in other similar populations in different settings be, new therapeutic goals and approaches based could be explored (instauration of early respiratory support in cases with increased NT-proBNP, pulmonary vasodilators to reduce RV pressure overload, avoiding epinephrine in cases with LVMD), in a disease without any current effective treatment.

In RSVB, prevention and early identification of infants at risk for severe disease in order to provide the best management options and potentially decrease morbidity are the major goals. Current guidelines recommend only clinical observation for this purpose in infants without known comorbidities [3]. However, most clinical scores for RSVB are not well validated and fail to predict outcomes $[39,40]$. Recently, the BROSJOD score, a validated clinical score for RSVB, has shown a strong capacity to predict the evolution in RSVB, but it is not generalizable due to the single-centre character of the study [26]. In this context, the identification of novel biomarkers with adequate predictive value for disease severity in RSVB is an area of increasing research interest. Neutrophins, cytokynes and leukotrienes are promising but not widely available for clinical practice [41]. Also, previous studies have tested cardiac troponin as prognostic marker but with inconsistent results $[9,10,12,13]$. The LVMD found in our population was only identifiable by TDI-echocardiography, suggesting that it was mild in most of our patients. Nevertheless, it does not mean that LVMD is inconsequential in RSVB. Remarkably, most patients with LVMD presented a severe disease at admission and most of them required PICU admission. Of note, we included non-ventilated infants at early stages of the disease (mean time of 2.76 (1.23) days after the initial symptoms), when the patients have not been admitted yet to PICU, increasing the prognostic value of our results. Therefore, assessing and understanding myocardial function in RSVB seems to be relevant.

Another interesting finding of our study was that NT-proBNP could be a useful biomarker for LVMD and subsequently outcomes in RSVB. Previous studies have also documented the correlation between LVTX and NT-proBNP plasmatic levels [42-44]. We explored the diagnostic accuracy of NT-proBNP to detect LVTX $>0.50$, which resulted high (AUC 0.91), with an optimal cut-off value of $1500 \mathrm{pg} / \mathrm{ml}$ (Se 0.80, Sp 0.95, PPV $0.80, \mathrm{NPV}$ 0.95). We also tested the benefit to add NTproBNP to the currently used clinical and laboratory data to assess outcomes in RSVB. Although the predictive models including age, BROSJOD score and RA presented a high predictive accuracy for PICU admission in our population, we observed that the addition of NTproBNP to the models increased significantly their predictive value, and that NTproBNP was the only independent factor within the analysed that predicted a severe course of the disease. Most cases of RSVB are mild forms manageable on an outpatient basis without need of laboratory exams. Nevertheless, many children sufficiently ill to require hospitalization will routinely have laboratory studies drawn. Adding NTproBNP determination to these studies could be useful, in order to identify high-risk patients that benefit of echocardiographic screening to PH or LVMD. Based on our results it might be reasonable to perform an echocardiogram in those patients with NT-proBNP levels $>1500 \mathrm{pg} / \mathrm{ml}$.

This work included some limitations. Our study was performed at a single center and with a relatively small size. We excluded irritable or unstable patients, where the technical difficulties due to respiratory comorbidity and the patient's worse tolerance to the evaluation could impact the results. The PICU admission criteria, and subsequently the definition of severe diseases in the study, are based on the protocol of our hospital, which can vary between different institutions. Therefore, a larger multicentre cohort study including irritable or unstable cases and with uniform PICU admission criteria may be needed for verification and generalization of our results. Finally the assessment of myocardial function and $\mathrm{PH}$ on echocardiography 
was not confirmed by an independent gold-standard method, such as cardiac magnetic resonance imaging or right heart catheterization, and therefore some patients could have been miss-classified.

5. Conclusions

LVMD secondary to adverse RV-LV interactions could be present in healthy infants with RSVB during early stages of the disease, negatively impacting the outcome. NTproBNP seems to be an adequate biomarker for LVMD and adding NTproBNP to current recommendations to assess outcomes in RSVB could help to detect early those cases that develop a severe illness. Future research is need in order to confirm these results and to design new therapeutic approach based on them.

Author Contributions: C onceptualization, RG.M; methodology, RG.M \& BF.I.; software, RG.M \& BF.I; validation, RG.M., BF.I., PR.A., CM.A., LL.S.; formal analysis, RG.M \& BF.I.; investigation, RG.M., PR.A. and CM.A..; resources, RG.M \& LL.S.; data curation, RG.M, CM.A. and PR.A.; writing-original draft preparation, RG.M.; writing-review and editing, RG.M., BF.I., PR.A., CM.A., LL.S..; visualization, RG-M. \& BF.I..; supervision, RG.M \& BF.I..; project administration, RG.M.

Funding: This research received no external funding.

Conflicts of Interest: The authors declare no conflict of interest.

References

1. Hasegawa K, Tsugawa Y, Brown DFM, Mansbach JM, Camargo CA. Trends in bronchiolitis hospitalizations in the United States, 2000-2009. Pediatrics. American Academy of Pediatrics; 2013 Jul;132(1):28-36.

2. Ghazaly M, Nadel S. Characteristics of children admitted to intensive care with acute bronchiolitis. Eur J Pediatr. 2018 Jun;177(6):913-20.

3. Ralston SL, Lieberthal AS, Meissner HC, Alverson BK, Baley JE, Gadomski AM, et al. Clinical practice guideline: the diagnosis, management, and prevention of bronchiolitis. Vol. 134, Pediatrics. American Academy of Pediatrics; 2014. pp. e1474-502.

4. Willson DF, Landrigan CP, Horn SD, Smout RJ. Complications in infants hospitalized for bronchiolitis or respiratory syncytial virus pneumonia. J Pediatr. 2003 Nov;143(5 Suppl):S142-9.

5. Tulloh RMR, Medrano-Lopez C, Checchia PA, Stapper C, Sumitomo N, Gorenflo M, et al. CHD and respiratory syncytial virus: global expert exchange recommendations. Cardiol Young. 2017 Oct;27(8):150421.

6. Fitzgerald D, Davis GM, Rohlicek C, Gottesman R. Quantifying pulmonary hypertension in ventilated infants with bronchiolitis: a pilot study. J Paediatr Child Health. 2001 Feb;37(1):64-6.

7. Bardi-Peti L, Ciofu EP. [Bronchiolitis and pulmonary hypertension]. Pneumologia. 2010 Apr;59(2):95-100.

8. Kimura D, McNamara IF, Wang J, Fowke JH, West AN, Philip R. Pulmonary hypertension during respiratory syncytial virus bronchiolitis: a risk factor for severity of illness. Cardiol Young. 2019 May 20;20:15.

9. Clark SJ, Eisenhut M, Sidaras D, Hancock SW, Newland P, Thorburn K. Myocardial injury in infants ventilated on the paediatric intensive care unit: a case control study. Crit Care. BioMed Central; 2006;10(5):R128.

10. Moynihan JA, Brown L, Sehra R, Checchia PA. Cardiac troponin I as a predictor of respiratory failure in children hospitalized with respiratory syncytial virus (RSV) infections: a pilot study. The American Journal of Emergency Medicine. 2003 Oct;21(6):479-82.

11. Thorburn K. Cardiac Troponin T levels and myocardial involvement in children with severe respiratory syncytial virus lung disease. Acta Paediatr. 2004 Jul;93(7):887-90. 
12. Thorburn K, Eisenhut M, Shauq A, Narayanswamy S, Burgess M. Right ventricular function in children with severe respiratory syncytial virus (RSV) bronchiolitis. Minerva Anestesiol. 2011 Jan;77(1):46-53.

13. Rodriguez-Gonzalez M, Benavente Fernández I, Castellano-Martinez A, Lechuga-Sancho AM, LubiánLópez SP. NT-proBNP plasma levels as biomarkers for pulmonary hypertension in healthy infants with respiratory syncytial virus infection. Biomarkers Med. 2019 Jun 3.

14. Driessen MMP, Meijboom FJ. Adverse ventricular-ventricular interactions in right ventricular pressure load: Insights from pediatric pulmonary hypertension versus pulmonary stenosis. Physiol Rep. 2016 Jun;4(11):e12833.

15. Burkett DA, Slorach C, Patel SS, Redington AN, Ivy DD, Mertens L, et al. Impact of Pulmonary Hemodynamics and Ventricular Interdependence on Left Ventricular Diastolic Function in Children With Pulmonary Hypertension. Circ Cardiovasc Imaging. 2016 Sep;9(9):e004612.

16. Motoji Y, Tanaka H, Fukuda Y, Sano H, Ryo K, Imanishi J, et al. Interdependence of right ventricular systolic function and left ventricular filling and its association with outcome for patients with pulmonary hypertension. Int J Cardiovasc Imaging. Springer Netherlands; 2015 Jan 23;31(4):691-8.

17. Pahl E, Gidding SS. Echocardiographic assessment of cardiac function during respiratory syncytial virus infection. Pediatrics. 1988 Jun;81(6):830-4.

18. Esposito S, Salice P, Bosis S, Ghiglia S, Tremolati E, Tagliabue C, et al. Altered cardiac rhythm in infants with bronchiolitis and respiratory syncytial virus infection. BMC Infect Dis. 2nd ed. BioMed Central; 2010 Oct 24;10(1):305.

19. Horter T, Nakstad B, Ashtari O, Solevåg AL. Right and left ventricular function in hospitalized children with respiratory syncytial virus infection. Infect Drug Resist. Dove Press; 2017;10:419-24.

20. Martinez-Rumayor A, Richards AM, Burnett JC, Januzzi JL Jr. Biology of the Natriuretic Peptides. Am J Cardiol. 2008 Feb;101(3):S3-S8.

21. Kate ten CA, Tibboel D, Kraemer US. B-type natriuretic peptide as a parameter for pulmonary hypertension in children. A systematic review. Eur J Pediatr. 2015 Aug 23;174(10):1267-75.

22. Takatsuki S, Wagner BD, Ivy DD. B-type natriuretic peptide and amino-terminal pro-B-type natriuretic peptide in pediatric patients with pulmonary arterial hypertension. Congenit Heart Dis. Blackwell Publishing Inc; 2012 May;7(3):259-67.

23. Hauser JA, Demyanets S, Rusai K, Goritschan C, Weber M, Panesar D, et al. Diagnostic performance and reference values of novel biomarkers of paediatric heart failure. Heart. 3rd ed. 2016 Sep 27;102(20):1633-9.

24. Mladosievicova B, Urbanova D, Radvanska E, Slavkovsky P, Simkova I. Role of NT-proBNP in detection of myocardial damage in childhood leukemia survivors treated with and without anthracyclines. Journal of Experimental \& Clinical Cancer Research; 2012 Oct 11;31(1):1-1.

25. Li Q, Cui C-Y, Zhang C, Guo S-Y, Zhang Q. On the changes of NT-proBNP level in children having undergone radical operation of tetralogy of Fallot and the clinical significance. Eur Rev Med Pharmacol Sci. 2015 Aug;19(16):3018-22.

26. Balaguer M, Alejandre C, Vila D, Esteban E, Carrasco JL, Cambra FJ, et al. Bronchiolitis Score of Sant Joan de Déu: BROSJOD Score, validation and usefulness. Pediatr Pulmonol. 2nd ed. 2017 Apr;52(4):533-9.

27. Lai WW, Geva T, Shirali GS, Frommelt PC, Humes RA, Brook MM, et al. Guidelines and Standards for Performance of a Pediatric Echocardiogram: A Report from the Task Force of the Pediatric Council of the American Society of Echocardiography. Journal of the American Society of Echocardiography. 2006 Dec;19(12):1413-30. 
28. Koestenberger M, Ravekes W, Everett AD, Stueger HP, Heinzl B, Gamillscheg A, et al. Right ventricular function in infants, children and adolescents: reference values of the tricuspid annular plane systolic excursion (TAPSE) in 640 healthy patients and calculation of z score values. J Am Soc Echocardiogr. 2009 Jun;22(6):715-9.

29. Tei C, Dujardin KS, Hodge DO, Bailey KR, McGoon MD, Tajik AJ, et al. Doppler echocardiographic index for assessment of global right ventricular function. Journal of the American Society of Echocardiography. 1996 Nov;9(6):838-47.

30. Koestenberger M, Apitz C, Abdul-Khaliq H, Hansmann G. Transthoracic echocardiography for the evaluation of children and adolescents with suspected or confirmed pulmonary hypertension. Expert consensus statement on the diagnosis and treatment of paediatric pulmonary hypertension. The European Paediatric Pulmonary Vascular Disease Network, endorsed by ISHLT and D6PK. Heart. BMJ Publishing Group Ltd and British Cardiovascular Society; 2016 May;102 Suppl 2(Suppl 2):ii14-22.

31. Habash S, Laser KT, Moosmann J, Reif R, Adler W, Glöckler M, et al. Normal values of the pulmonary artery acceleration time (PAAT) and the right ventricular ejection time (RVET) in children and adolescents and the impact of the PAAT/RVET-index in the assessment of pulmonary hypertension. Int J Cardiovasc Imaging. Springer Netherlands; 2019 Feb;35(2):295-306.

32. Abraham S, Weismann CG. Left Ventricular End-Systolic Eccentricity Index for Assessment of Pulmonary Hypertension in Infants. Echocardiography. 2016 Jan 16;33(6):910-5.

33. Cui W, Roberson DA. Left ventricular Tei index in children: comparison of tissue Doppler imaging, pulsed wave Doppler, and M-mode echocardiography normal values. J Am Soc Echocardiogr. 2006 Dec;19(12):143845.

34. DeLong ER, DeLong DM, Clarke-Pearson DL. Comparing the areas under two or more correlated receiver operating characteristic curves: a nonparametric approach. Biometrics. 1988 Sep;44(3):837-45.

35. Koo TK, Li MY. A Guideline of Selecting and Reporting Intraclass Correlation Coefficients for Reliability Research. J Chiropr Med. 2016 Jun;15(2):155-63.

36. Cui W, Roberson DA, Chen Z, Madronero LF, Cuneo BF. Systolic and diastolic time intervals measured from Doppler tissue imaging: normal values and Z-score tables, and effects of age, heart rate, and body surface area. J Am Soc Echocardiogr. 2008 Apr;21(4):361-70.

37. Burkett DA, Slorach C, Patel SS, Redington AN, Ivy DD, Mertens L, et al. Left Ventricular Myocardial Function in Children With Pulmonary Hypertension: Relation to Right Ventricular Performance and Hemodynamics. Circ Cardiovasc Imaging. American Heart Association, Inc; 2015 Aug;8(8):e003260-19.

38. Haeck MLA, Höke U, Marsan NA, Holman ER, Wolterbeek R, Bax JJ, et al. Impact of right ventricular dyssynchrony on left ventricular performance in patients with pulmonary hypertension. Int J Cardiovasc Imaging. Springer Netherlands; 2014 Feb 4;30(4):713-20.

39. Bekhof J, Reimink R, Brand PLP. Systematic review: Insufficient validation of clinical scores for the assessment of acute dyspnoea in wheezing children. Paediatric Respiratory Reviews. 2014 Mar;15(1):98-112.

40. Destino L, Weisgerber MC, Soung P, Bakalarski D, Yan K, Rehborg R, et al. Validity of respiratory scores in bronchiolitis. Hosp Pediatr. 2012 Oct;2(4):202-9.

41. Brown PM, Schneeberger DL, Piedimonte G. Biomarkers of respiratory syncytial virus (RSV) infection: specific neutrophil and cytokine levels provide increased accuracy in predicting disease severity. Paediatric Respiratory Reviews. 2015 Sep;16(4):232-40.

42. Takeuchi D, Saji T, Takatsuki S, Fujiwara M. Abnormal tissue doppler images are associated with elevated plasma brain natriuretic peptide and increased oxidative stress in acute Kawasaki disease. Circ J. 2007 Mar;71(3):357-62. 
43. Ozde C, Dogru M, Ozde Ş, Kayapinar O, Kaya A, Korkmaz A. Subclinical right ventricular dysfunction in intermittent and persistent mildly asthmatic children on tissue Doppler echocardiography and serum NTproBNP: Observational study. Pediatr Int. John Wiley \& Sons, Ltd (10.1111); 2018 Nov;60(11):1024-32.

44. Mikkelsen KV, Møller JE, Bie P, Ryde H, Videbaek L, Haghfelt T. Tei index and neurohormonal activation in patients with incident heart failure: serial changes and prognostic value. European Journal of Heart Failure. John Wiley \& Sons, Ltd; 2006 Oct;8(6):599-608.

(C) 2019 by the authors. Submitted for possible open access publication under the terms and conditions of the Creative C 